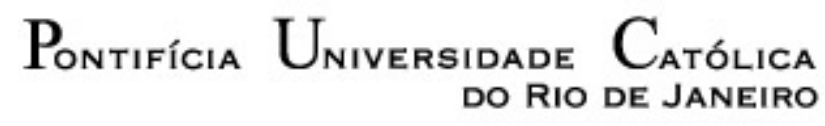

DO RIO DE JANEIRO

Felipe Prado Loureiro

Modelagem Acústica no Domínio da Transformada Wavelet

Dissertação de Mestrado

Dissertação apresentada como requisito parcial para obtenção do título de Mestre pelo Programa de Pós-Graduação em Engenharia Elétrica da PUC-Rio.

Orientador: Paulo Léo Manassi Osório 


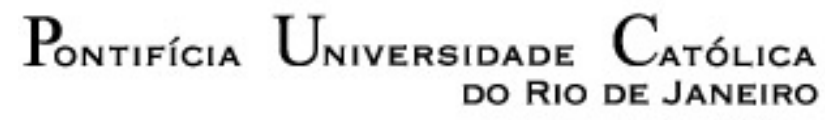

DO RIO DE JANEIRO

Felipe Prado Loureiro

\title{
Modelagem Acústica no Domínio da \\ Transformada Wavelet
}

\begin{abstract}
Dissertação de Mestrado apresentada como requisito parcial para obtenção do grau de Mestre pelo Programa de Pós-Graduação em Engenharia Elétrica do Departamento de Engenharia Elétrica do Centro Técnico Científico da PUC-Rio. Aprovada pela Comissão Examinadora abaixo assinada.
\end{abstract}

Dr. Paulo Léo Manassi Osório

Orientador

Departamento de Engenharia Elétrica - PUC-Rio

Dr. Djalma Manoel Soares Filho PETROBRAS

Dr. Marcos Azevedo da Silveira Departamento de Engenharia Elétrica - PUC-Rio

Dr. Marcos Antônio Gallotti Guimarães PETROBRAS

Dr. Mauro Speranza Neto Departamento de Engenharia Mecânica - PUC-Rio

Prof. José Eugenio Leal Coordenador Setorial do Centro

Técnico Científico - PUC-Rio

Rio de Janeiro, 16 de março de 2004 
Todos os direitos reservados. É proibida a reprodução total ou parcial do trabalho sem autorização da universidade, do autor e do orientador.

\section{Felipe Prado Loureiro}

Graduou-se em Engenharia de Controle e Automação pela Pontifícia Universidade Católica do Rio de Janeiro em 2001. Prestou serviços nesta área no setor de pesquisa da empresa de engenharia offshore DSND Consub SA sob regime de estágio. Participou do $8^{\circ}$ Congresso Internacional da Sociedade Brasileira de Geofísica em setembro de 2003.

Ficha Catalográfica

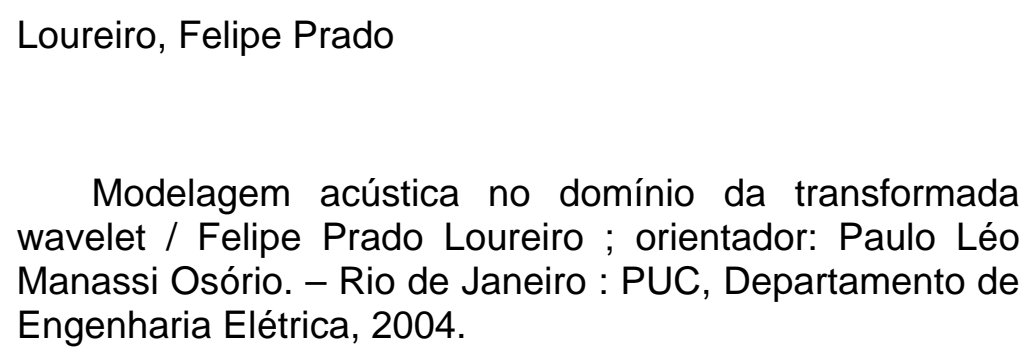

Modelagem acústica no domínio da transformada wavelet / Felipe Prado Loureiro ; orientador: Paulo Léo Manassi Osório. - Rio de Janeiro : PUC, Departamento de Engenharia Elétrica, 2004.

72 f. ; $30 \mathrm{~cm}$

Dissertação (mestrado) - Pontifícia Universidade Católica do Rio de Janeiro, Departamento de Engenharia Elétrica.

Inclui referências bibliográficas.

1. Engenharia elétrica - Teses. 2. Modelagem sísmica. 3. Migração sísmica. 4. Transformada wavelet. 5. Diferenciação no domínio da transformada walvelet. 6. Processamento paralelo. I. Osório, Paulo Léo Manassi. II. Pontifícia Universidade Católica do Rio de Janeiro. Departamento de Engenharia Elétrica. III. Título. 
Para meus pais, Pompeu e Mirtes,

pelo suporte e encorajamento. 


\section{Agradecimentos}

Ao meu orientador Paulo Léo Manassi Osório, pela proposição do tema de minha dissertação e pelo estímulo em sua conclusão, que motivou minha vontade mesmo quando esta parecia desaparecer.

Aos professores Moisés Henrique Szwarcman e Mauro Speranza Neto, pela demonstração de confiança em minha capacidade quando do término de minha graduação e indicação ao programa de pós-graduação.

À PETROBRAS e à PUC-Rio, pelos auxílios concedidos, sem os quais este trabalho não poderia ter sido realizado.

Aos meus pais, pelo apoio incondicional e irrestrito oferecido no decorrer de minha jornada acadêmica. À minha família, pelo conforto de sua presença e pelo sossego de sua ausência.

Ao colega e amigo Alexandre de Lima Spinola, pelo exemplo de dedicação simultânea ao trabalho, aos estudos e à vida pessoal.

Aos colegas pós-graduandos Marcílio C. de Matos, Gustavo P. de Castro e Marco Antonio C. Santos, pelas discussões e conselhos sobre os diversos temas técnicos em torno da pesquisa para esta dissertação, em especial ao último, por suas maiores presença e insistência. Aos velhos amigos Cesar Matos, Fernando Esberard, Leonardo Wrigg, Rafael Gimenez, Renato Oliveira e Rodrigo Lessa pelos momentos de descontração e por posarem como modelos da diversidade de temperamentos, existente mesmo em um grupo tão unido.

Àqueles amores e desamores, que ficaram pouco tempo e deixaram grandes marcas, por me terem feito aprender sozinho que existem males que vêm para bem.

Aos professores que participaram da banca examinadora. 


\section{Resumo}

Loureiro, Felipe Prado; Osório, Paulo Léo Manassi. Modelagem Acústica no Domínio da Transformada Wavelet. Rio de Janeiro, 2004. 72p. Dissertação de Mestrado - Departamento de Engenharia Elétrica, Pontifícia Universidade Católica do Rio de Janeiro.

O processamento de sinais sísmicos é peça chave na exploração petrolífera. O caminho entre aquisição de dados e interpretação sísmica é composto por uma trilha de processos interdependentes, entre eles os processos de modelagem e migração. A dissertação apresenta a composição de um algoritmo de modelagem acústica 2D no domínio da transformada wavelet a partir de ferramentas próprias e outras já existentes na literatura. São estabelecidas as aproximações necessárias à solução em meios heterogêneos e à independência entre os subdomínios de processamento. Esta independência possibilita a exploração de técnicas de processamento paralelo. Através de exemplos, seu desempenho é avaliado com comparações à solução via diferenças finitas. Estas soluções são ainda submetidas ao mesmo processo de migração baseado em um terceiro modo de solução.

\section{Palavras-chave}

Modelagem sísmica; migração sísmica; transformada wavelet; diferenciação no domínio da transformada wavelet; processamento paralelo. 


\section{Abstract}

Loureiro, Felipe Prado; Osório, Paulo Léo Manassi (Advisor). Acoustic Modeling in the Wavelet Transform Domain. Rio de Janeiro, 2004. 72p. MSc. Dissertation - Departamento de Engenharia Elétrica, Pontifícia Universidade Católica do Rio de Janeiro.

Seismic signal processing is a key step to oil exploration. The path between data acquisition and seismic interpretation is composed by a sequence of interdependent processes, among which are modeling and migration processes. A 2D acoustic modeling algorithm in wavelet Transform domain, based on custom tools and tools already made known in literature is presented. Approximations necessary for the solution in inhomogeneous media and for complete independence between processing subspaces are established. Such independence allows exploration of parallel processing techniques. Throughout examples, performance is evaluated in comparison to finite-difference solution. These solutions are further processed by a migration technique based in yet another solution method.

\section{Keywords}

Seismic modeling; seismic migration; wavelet transform; differentiation in the wavelet transform domain; parallel processing. 


\section{Sumário}

1 Introdução

2 Modelagem e migração sísmicas

2.1. Introdução

17

2.2. Propagação de ondas acústicas 17

2.3. Métodos clássicos de solução da equação de onda 18

2.3.1. Diferenças finitas 18

2.3.2. Pseudo-espectral 20

2.4. Outros métodos de solução da equação da onda 21

2.4.1. Rotação de Fase Mais Interpolação 21

2.4.2. Split-step 21

2.4.3. Transformada wavelet 22

3 Modelagem acústica por Transformada wavelet 23

3.1. Transformada wavelet 23

3.1.1. Transformada wavelet discreta 25

3.1.2. Transformada wavelet discreta por banco de filtros 28

3.1.3. Transformada wavelet discreta em duas dimensões 30

3.2. Diferenciação no domínio Wavelet 34

3.3. Solução para meios homogêneos 36

3.4. Solução para meios heterogêneos $\quad 37$

3.4.1. Construção dos campos de velocidades 37

3.5. Tratamento de borda/limite $\quad 39$

3.6. Processos, otimizações e fluxograma 40

3.6.1. Inclusão do termo forçado 41

3.6.2. Reconstrução parcial 42

3.6.3. Descrição do algoritmo 44

$\begin{array}{ll}4 \text { Resultados } & 48\end{array}$

4.1. Desempenho do algoritmo proposto 48 
4.1.1. Precisão dos sismogramas

5 Conclusões

6 Referências bibliográficas 


\section{Lista de figuras}

Figura 1: A função periódica senoidal e a função transiente wavelet Daubechies com 10 momentos nulos 24

Figura 2: Decomposição wavelet 1D por banco de filtros 29

Figura 3: Bandas de freqüência na árvore de decomposição 30

Figura 4: Arvore de reconstrução (síntese) por banco de duas bandas 30 Figura 5: Partição de coeficientes wavelet 1D. Note o aumento do número (proporcional ao tamanho desenhado) de coeficientes resultantes devido aos resíduos da convolução. $\quad 31$

Figura 6: Subespaços wavelet na forma de decomposição padrão. 32 Figura 7: Análise wavelet 2D em um nível. O modo alternado é derivado das sucessivas análises dos subespaços $A_{j}$. 32

Figura 8: Partição dos coeficientes 2D, modo alternado e esparso. $\quad 33$ Figura 9: Construção dos modelos de velocidades para cada subespaço. Semelhante ao processo de transformada wavelet, porém somente o resultado da aproximação é considerado.

Figura 10: Construção dos modelos de velocidades para cada subespaço. Resultado da partição em dois níveis de decomposição.

Figura 11: Bordas de absorção. (a) matriz para diferenças finitas; (b) subespaços da matriz para transformada wavelet.

Figura 12: Fluxograma do algoritmo de modelagem via transformada wavelet

Figura 13: Sub-rotinas utilizadas no algoritmo de modelagem por transformada wavelet, mostradas na Figura 12

Figura 14: Exemplo 1. (a) o sinal $S(x, z)=\operatorname{sen}(4 x)+\operatorname{sen}(7 z)$; (b) o resultado da operação usando transformada wavelet; (c) o resultado da operação usando diferenças finitas; (d) erro presente em (b); (e) erro presente em (c); (f) faixas selecionadas em (a), (b) e (c); (g) faixas selecionadas em (d) e (e).

Figura 15: Exemplo 2. Construção do modelo de velocidades para os subespaços wavelet. Erros no posicionamento da segunda camada 
devido à decimação do campo de velocidades: (a) perfil de velocidades; (b) sismograma obtido por diferenças finitas (padrão); (c) sismograma obtido por wavelets e decimação direta; (d) sismograma obtido por wavelets e decimação após a filtragem proposta; (e) Comparação entre as amplitudes ao longo das linhas assinaladas em (b), (c) e (d).

Figura 16: Exemplo 3. (a) perfil abrupto de velocidades. (b) sismograma obtido por diferenças finitas. (c) sismograma obtido por wavelets.

Figura 17: Exemplo 3. (a) suavização do perfil de velocidades da figura 16. (b) sismograma obtido por diferenças finitas. (c) sismograma obtido por wavelets.

Figura 18: Comparação entre as amplitudes ao longo das linhas assinaladas nos sismogramas das figuras 16 e 17: (a) detalhe entre os instantes 1,0s e 1,3s; (b) detalhe entre os instantes 2,2s e 2,5s.

Figura 19: Exemplo 4. (a) modelo utilizado; (b) sismograma obtido por diferenças finitas; (c) sismograma obtido por transformada wavelet.

Figura 20: Exemplo 4. (a), (b) e (c) instantâneos (estados) do campo acústico em $0,16 \mathrm{~s}, 0,18$ s e $0,22 \mathrm{~s}$, respectivamente, obtidos pelo método wavelet.

Figura 21: Exemplo 4. (a), (b) e (c) ampliação dos traços escolhidos na figura 19 (b) e (c).

Figura 22: Exemplo 4. (a) perfil migrado do sismograma da figura 19 (b); (b) perfil migrado do sismograma da figura 19 (c).

Figura 23: Exemplo 4. Traço escolhido pela linha vertical cheia nas figuras 22 (a) e (b).

Figura 24: Exemplo 4. Detalhe marcado pela região cinza na figura 23. 57 Figura 25: Exemplo 5. (a) modelo utilizado; (b) sismograma obtido por diferenças finitas; (c) sismograma obtido por transformada wavelet; (d), (e) e (f) ampliação dos traços escolhidos em (b) e (c).

Figura 26: Exemplo 6. Modelo utilizado, com duas interfaces inclinadas. 61 Figura 27: Exemplo 6. Sismogramas obtidos. (a) por diferenças finitas; (b), (c) e (d) por transformada wavelet com as funções db2, db4 e db10 respectivamente;

Figura 28: Exemplo 6. (a) traço escolhido nas figuras 27 (a), (b), (c) e (d); 
(b) ampliação da região escolhida em (a).

Figura 29: Exemplo 6. (a) a (d) modelos migrados a partir dos sismogramas das figuras 27 (a) a (d).

Figura 30: Exemplo 6. Traço escolhido pela linha vertical nas figuras 29 (a) a (d).

Figura 31: Exemplo 6. Ampliação do detalhe marcado em cinza na figura 30.

Figura 32: Exemplo 7. (a) modelo utilizado; (b) sismograma obtido por diferenças finitas; (c) sismograma obtido por transformada wavelet com a função db4; (d) traço escolhido em (b) e (c).

Figura 33: Exemplo 7. (a) e (b) Modelos migrados a partir dos sismogramas das figuras 32 (b) e (c). 66

Figura 34: Exemplo 7. Traço escolhido nas figuras 33 (a) e (b). $\quad 66$ 
Dois importantes fatos, nesta vida, saltam aos olhos. Primeiro, que cada um de nós sofre inevitavelmente derrotas temporárias, de formas diferentes, nas ocasiões mais diversas. Segundo, que cada adversidade traz consigo a semente de um benefício equivalente. Ainda não encontrei homem algum bem-sucedido na vida que não houvesse antes sofrido derrotas temporárias. Toda vez que um homem supera os reveses, torna-se mental $e$ espiritualmente mais forte. É assim que aprendemos o que devemos à grande lição da adversidade.

Andrew Carnegie 